El cuento y el álbum: (in)visibilidad femenina en la literatura infantil y juvenil vasca del siglo XXI

\title{
EL CUENTO Y EL ÁLBUM: (IN)VISIBILIDAD FEMENINA EN LA LITERATURA INFANTIL Y JUVENIL VASCA DEL SIGLO XXI
}

Xabier ETXANIZ

$\mathrm{UPV} / \mathrm{EHU}$

xabier.etxaniz@ehu.eus

\section{Resumen:}

En la Literatura Infantil y Juvenil (LIJ) vasca se ha dado un incremento de la presencia de autoras y protagonistas femeninas. Esta feminización de la LIJ se ha dado en todos los ámbitos pero, sobre todo, en las obras premiadas y en los álbumes. Analizamos no solo el incremento cuantitativo de las autoras -escritoras e ilustradoras-, sino también la imagen de la mujer que se ofrece en las nuevas obras.

Palabras clave: Literatura infantil y juvenil, feminismo, obras premiadas.

\section{LE CONTE ET L'ALBUM: (IN)VISIBILITÉ FÉMININE DANS LA LITTÉRATURE BASQUE D'ENFANCE ET POUR LA JEUNESSE DU XXI ${ }^{\mathrm{e}}$ SIÈCLE}

\section{Résumé:}

Dans la littérature basque pour enfants et jeunes la présence des femmes écrivains et de protagonistes féminines a augmenté. Cette féminisation de la littérature pour les enfants et les jeunes s'est produite dans tous les domaines, mais surtout dans les œuvres et les albums primés. Nous 


\title{
Xabier Etxaniz
}

analysons non seulement l'augmentation quantitative des femmes, écrivains et illustrateurs, mais également l'image de la femme proposée dans les nouvelles œuvres.

Mots-clés: Littérature d'enfants et jeunesse. Féminisation. Titres gagnants.

\section{TALES AND PICTURE BOOKS: FEMALE'S (IN) VISIBILITY IN CHILDREN'S AND YOUNG PEOPLE'S BASQUE LITERATURE FROM THE TWENTY-FIRST CENTURY}

\begin{abstract}
:
In Basque Literature for Children and Young People has been an increase of the female presence in both authors and protagonists. This feminization of the Literature for Children and Young People has occurred in all areas but, above all, in the awarded works and albums. We analyze not only the quantitative increase of the authors -writers and illustrators-, but also the image of the woman that is offered in the new works.

Keywords: Children's and Young People, feminization, awarded works.

En los últimos años, al hablar de la situación de la LIJ vasca hemos comentado más de una vez su creciente feminización (Etxaniz, 2013, 2017). Cuando hablamos de ello, estamos haciendo hincapié en el incremento de creadoras (escritoras e ilustradoras principalmente, pero también traductoras) así como el aumento de la presencia de personajes femeninos y la disminución de estereotipos discriminatorios en función del sexo (García Villanueva y Hernández Ramírez, 2016), puesto que la Literatura Infantil y Juvenil (LIJ), como elemento educativo, puede tener gran peso como modelo a identificarse por parte de los lectores, tal y como indica A. Moreno (1986):
\end{abstract}

La imagen que se da a los alumnos de la mujer y el hombre a través de los contenidos de la enseñanza, contribuye poderosamente a conformar su yo social, sus pautas diferenciales de comportamiento, el modelo con el que deben identificarse para ser «más mujer»o «más hombre» y les informa, a la vez, de la diferente valoración que nuestra sociedad hace de los individuos de cada sexo (pp. 29-30). 


\section{El cuento y el álbum: (in)visibilidad femenina en la literatura infantil y juvenil vasca del siglo XXI}

No entraremos ahora en el análisis de los receptores, de las personas lectoras, los porcentajes de niños y niñas que leen, el papel de los mediadores... todos los estudios realizados hasta ahora, así como los resultados de éxito escolar o abandono («La educación escolar en Euskadi: informe 2015-2017» del Consejo Escolar de Euskadi, http://www.consejoescolardeeuskadi.hezkuntza.net/ web/guest) demuestran que en Euskadi las chicas son más lectoras, obtienen mejores calificaciones y tienen una tasa de abandono escolar, 5,5\% inferior al de los chicos, 8,5\%.

Nuestra intención a lo largo de este estudio es analizar cómo ha ido aumentando la presencia de mujeres en el proceso creativo de la LIJ vasca y cómo, como consecuencia de este hecho, ese mundo que tradicionalmente ha sido mayoritariamente masculino se va feminizando cada vez más; este fenómeno se ha dado en los últimos años y, principalmente, en el ámbito de los álbumes literarios.

En la literatura infantil y juvenil en castellano, por ejemplo, la mayoría de autoras son mujeres (Freixas, 2009). Tal vez, como indica Celia Vazquez, (2010, p. 27) porque

\footnotetext{
la preferencia de la mujer por la producción infantil podría estar relacionada con la propia marginalidad del género dentro del conjunto de la literatura adulta. Precisamente esta marginalidad posibilitaría la expresión de su particular marginalidad femenina.
}

Frente a ello, en el ámbito de la LIJ vasca, el hecho de que dicha literatura surja en la década de los 80 y que tenga una gran presencia en el mercado editorial (un 25\% en sus inicios y ahora casi un 20\%), así como la importancia que se da a dicho género en la literatura vasca (prácticamente casi todos los autores de literatura para adultos de la década de los 80 escriben también LIJ), puede ser la causa de que, en contra de otras literaturas, en sus inicios la LIJ vasca no fuese muy femenina. Prueba de ello son los primeros catálogos de autores e ilustradores de LIJ vasca que se publicaron en 1997.

En el catálogo de escritores (1997b) había un total de 22 personas de las cuales 4 eran escritoras. En cuanto a las ilustradoras estas también eran 4 de un total de 24 personas que aparecían en el catálogo (1997a). Como se puede ver la presencia de mujeres es claramente inferior a la de los hombres. Otro tanto podríamos decir de las editoras; las principales editoriales vascas de la década de los 80, Elkar y Erein, tienen como editores de LIJ a dos hombres, Joxan Ormazabal y Anjel Lertxundi. Posteriormente, otras editoriales como Pamiela, Aizkorri, Zubia,... también tendrán a editores masculinos en sus colecciones de LIJ. Tan solo las editoriales Giltza-Edebé, Ibaizabal y Gero- 


\section{Xabier Etxaniz}

Mensajero tienen a mujeres al frente de las colecciones de LIJ.

Ahora bien, en los últimos años ha habido una serie de cambios y los principales son consecuencia, además, de los posibles avances que se han dado en la sociedad, de la mayor presencia de mujeres escritoras en la literatura vasca (no solo en la LIJ) y la incorporación al mundo editorial de las generaciones de personas que han salido de la facultad de Bellas Artes de la Universidad del País Vasco (UPV/EHU), facultad con un alumnado mayoritariamente femenino.

Concretamente en la LIJ se ha dado un aumento significativo de autoras e ilustradoras y, lo que es más importante, del peso que estas nuevas creadoras tienen dentro del sistema literario. Así si analizamos, por ejemplo, los principales premios de LIJ en el País Vasco, podemos apreciar que efectivamente en la LIJ vasca se ha dado una mayor feminización.

Así, por ejemplo, entre los autores galardonados con el premio Lizardi desde el año 2000 (14) nos encontramos con 8 mujeres y 6 hombres. Pero si analizáramos los datos desde el año 2010 el resultado es de 6 mujeres y un hombre (Anexo I).

Igualmente, la relación de autores galardonados con el premio Euskadi de LIJ desde el año 2000 (16), 11 hombres y 5 mujeres; y desde el año 2010: 9 (4 hombres y 5 mujeres), refleja dicha evolución (Anexo II); y la de personas galardonadas con el premio Euskadi de ilustración, premio creado en 2009, es de 9 personas (4 hombres y 5 mujeres, una de ellas, Elena Odriozola, dos veces) (Anexo III).

Ahora bien, esta presencia cada vez más femenina en la producción literaria, se acentúa en el ámbito de los álbumes. De las 12 obras galardonadas con el premio Etxepare que concede desde 2006 la mancomunidad de municipios navarros al mejor álbum en euskera (Anexo IV) dos autores repiten galardón (Pello Añora y Jokin Mitxelena por una parte y Leire Salaberria por otra); así tenemos que entre los galardonados hay 6 escritores y 4 escritoras, y junto a ellos 1 ilustrador y 9 ilustradoras.

Es decir, de los 20 autores y autoras de estas obras, un tercio son hombres y dos tercios mujeres. Una diferencia mucho mayor (1-9) si tenemos en cuenta solo la ilustración. 
El cuento y el álbum: (in)visibilidad femenina en la literatura infantil y juvenil vasca del siglo XXI

\section{Disminución de los estereotipos}

Estos cambios que apreciamos en la presencia de creadoras, también deberían ir acompañados de una mayor presencia de personajes femeninos o de una disminución de los estereotipos sexistas en la LIJ y en el mundo del álbum en particular, porque tal y como indica Mariasun Landa en una entrevista realizada por Karolina Almagia en la revista Emakunde (Almagia, 2009, p. 19), no se concibe la literatura sólo como un «pretexto» para inculcar valores:

Estos van incluidos en la forma de ver el mundo del autor. En mi caso soy muy sensible a la cuestión de la igualdad entre hombres y mujeres, a la lucha contra el sexismo $\mathrm{y}$, como consecuencia, pienso que eso se ha reflejado en mis narraciones como algo natural.

Y esto que indica Mariasun Landa lo podríamos ampliar a todo o a la mayoría del abanico de escritoras e ilustradoras vascas. Más aún si tenemos en cuenta la sensibilidad cada vez mayor por parte de la sociedad y el peso que tiene la lectura literaria, y es que, como indica Schwickart (1999, p. 123), "no debemos dejar de lado la actividad de la lectura, pues es aquí donde la literatura se realiza como praxis y actúa sobre el mundo al obrar sobre sus lectores".

Así, por ejemplo, es notoria la mayor presencia de protagonistas principales femeninas en las obras galardonadas con el premio Etxepare (72,7\%), frente a otras épocas anteriores (15,7\% de protagonistas principales femeninas en la década de los 80) o al conjunto de obras creadas en euskera a lo largo del año 2013 (32,7\%) (Fernández, 2017).

PROTAGONISTA PRINCIPAL

Etxepare Década 80 Año 2013

$\begin{array}{lccc}\text { Masculino } & 18,2 \% & 58,6 \% & 34,5 \% \\ \text { Femenino } & 72,7 \% & 15,7 \% & 32,7 \% \\ \text { Mixto-Otros } & 9,1 \% & 25,7 \% & 32,8 \%\end{array}$

Como se puede apreciar, si ya en las obras publicadas en el año 2013 se ve una equiparación en relación a la característica de protagonista principal de las obras, en las galardonadas con el premio Etxepare hay una gran mayoría de personajes femeninos, cuadruplicando el de los masculinos. 


\section{Xabier Etxaniz}

Ahora bien, como hemos indicado otras veces no solo debemos fijarnos en la cantidad de personajes o autores masculinos y femeninos al analizar la evolución que se está dando en la literatura. Principalmente deberemos analizar cómo se presentan dichos personajes. Hace casi medio siglo, según citan Colomer y Olid (2009) la influyente selección Little Miss Muffet Fights Back acordó unos criterios para incorporar libros no discriminatorios -o menos discriminatorios- en las selecciones de las bibliotecas y escuelas norteamericanas. Entre estos criterios podemos destacar:

1. Libros con mujeres y niñas activas e interesadas en su profesión o sus aventuras

2. Personajes femeninos con características de personalidad positivas, frente a las caracterizaciones tradicionales.

3. Retratos de mujeres que demuestren ambición y capacidad de tomar sus propias decisiones.

Si analizamos las obras galardonadas con el premio Etxepare, podemos ver, por ejemplo, que con respecto a las características físicas de los protagonistas (fuerza, debilidad y hermosura), hay una clara evolución en comparación con las obras de la década de los 80 o con los libros publicados en el año 2013.

Características físicas de los y las protagonistas.

$\begin{array}{lccc}\text { FUERZA } & \text { Etxepare } & \text { Década } 80 & \text { Año } 2013 \\ \text { Masculino } & 12 & 36 & 11 \\ \text { Femenino } & 9 & 4 & 4\end{array}$

\section{DEBILIDAD}

Masculino

1

0

0

Femenino

12

2

3

HERMOSURA

Masculino

10

3

9

Femenino

13

22 
El cuento y el álbum: (in)visibilidad femenina en la literatura infantil y juvenil vasca del siglo XXI

Como se puede apreciar, aunque en lo relativo a algunas cualidades (ser una persona fuerte o hermosa) se han dado pasos hacia la normalización, y en mayor medida que la media de las obras publicadas en esta época (concretamente en el año 2013), no podemos decir lo mismo respecto a la debilidad, una característica que sigue siendo femenina incluso en las obra del premio Etxepare.

Ahora bien, las características que mejor demuestran la evolución que se ha dado en la LIJ vasca de estos últimos años son las psicológicas. Concretamente, en los álbumes analizados, vemos que las niñas protagonistas son más activas, toman sus decisiones y juegan con otros niños sin ningún tipo de complejos.

En Euria ari duenean, Leire Salaberria nos cuenta la historia de una niña que disfruta con la lluvia, que le gusta que llueva y es feliz mojándose. La autora nos presenta una niña activa, soñadora, aventurera. Una chica que juega con sus amigos en los pozos de agua, que disfruta de la lluvia paseando con su perro (Fig. 1).

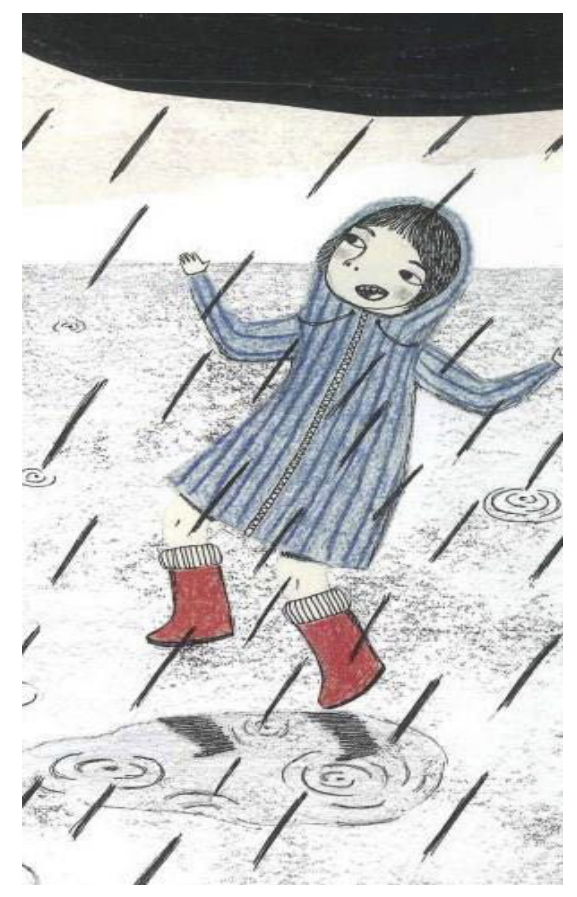

Fig. 1. Leire Salaberria. Euria ari duenean, Pamiela, Pamplona, 2011.

La niña de Hara!, obra escrita e ilustrada por Miren Asiain, es una niña activa que va sola a la piscina y cuando sale se marcha con su abuela a dar una vuelta, se nos ofrece una imagen de una niña segura, atrevida... pero también la abuela (ambas son las protagonistas de este álbum 


\section{Xabier Etxaniz}

en el que se nos narra la relación que mantienen ambas durante dos días) se presenta como una persona activa, que lleva a su nieta por el bosque, van al cine, hacen las compras juntas o le prepara un vestido sorpresa. Una abuela que no para y una nieta atrevida que disfruta con su compañía porque, esta abuela ha roto con los estereotipos existentes. "El papel de la abuela resulta especialmente estereotipado. Ignorando que las abuelas con nietos de cinco años son jóvenes de cincuenta, los cuentos muestran a viejas a las puertas de la muerte" (Turin, 2009, p. 31) (Fig.2).

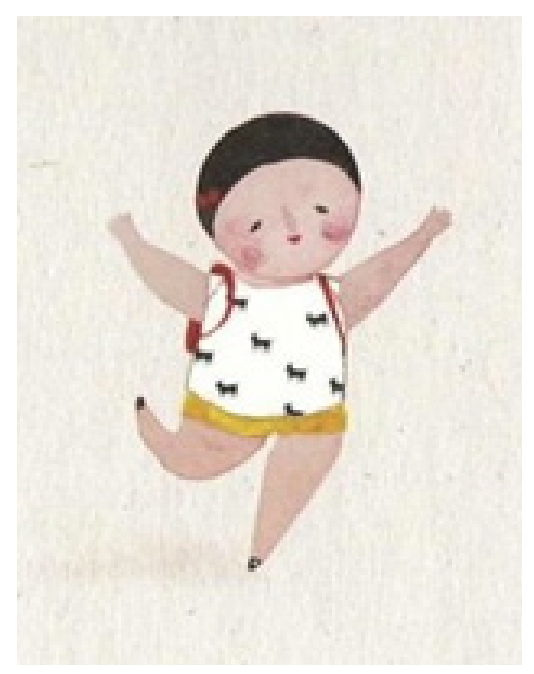

Fig. 2. Miren Asiain Lora. Hara!, Pamiela, Pamplona, 2012

Otro tanto podríamos decir de la niña protagonista del álbum Alex, nire laguna (Mi amigo Alex), una niña que juega con los niños después de haber superado su incomunicación, su falta de amistades o relaciones con los demás, una ausencia que suple con una amigo imaginario -Alex, que es quien da nombre al libro-. La protagonista del libro logra superar su timidez, sus dificultades y finalmente ya no necesita a ningún amigo imaginario para poder salir adelante (Fig. 3). 
El cuento y el álbum: (in)visibilidad femenina en la literatura infantil y juvenil vasca del siglo XXI

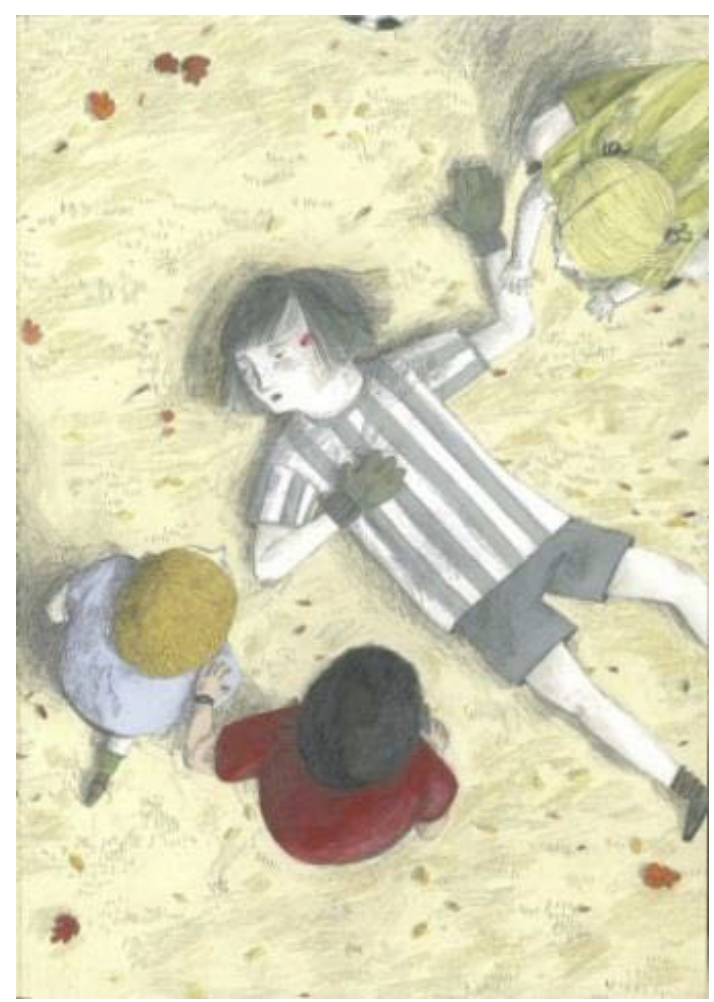

Fig. 3. Maite Gurrutxaga. Alex nire laguna. Pamiela, Pamplona, 2010.

Los personajes femeninos tienen fuerza por sí mismos, son libres e intentan romper estereotipos, tal y como indica Arrata Egaña (in Almagia, 2009, p. 21) cuando dice que sus personajes son capaces de romper con los impedimentos existentes, son autónomas y saben cuidarse ellas solas. Así, en Zerua gris dago es la niña que anda sola por la nieve quien arroja una bola de nieve a un chico provocándole para que jueguen ellos dos en la soledad del paisaje nevado. La mirada pícara de la niña muestra la desvergüenza, el atrevimiento y el valor que tiene (Fig. 4) porque nos encontramos en un escenario en el que no hay casi movimiento ni personajes. Un niño camina solo por la nieve, no hay nadie en la calle. De repente ve a lo lejos a una chica que le mira y que, cuando menos se lo espera, le arroja una bola de nieve para comenzar un juego, una relación entre ellos durante un breve momento con la esperanza de que al día siguiente también se le aparezca esa niña y puedan seguir jugando. 


\section{Xabier Etxaniz}

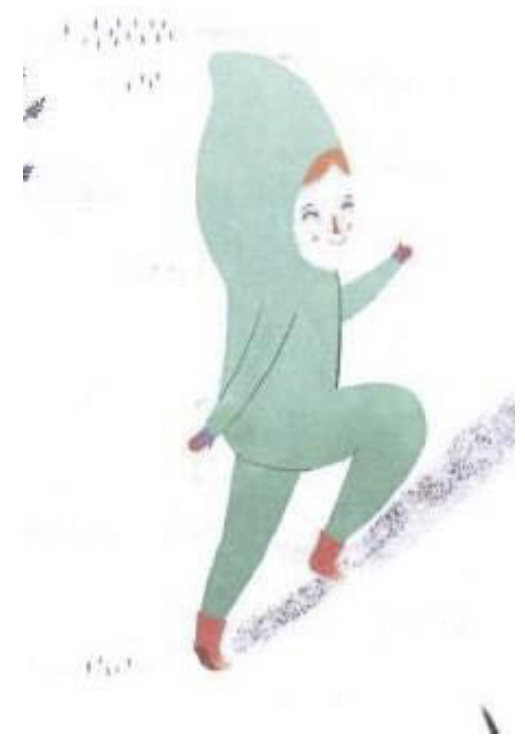

Fig. 4. Isaak Martinez. Zerua gris dago. Pamiela, Pamplona, 2016.

Junto a estas niñas también están las sensibles e inocentes como la narradora de la historia Gerrak ez du izenik ambientada en la guerra civil, concretamente el día en que la villa de Durango fue bombardeada por la aviación italiana y en la que la niña protagonista de la narración, Mirentxu, decide no hablar puesto que tal y como indica un refrán vasco lo que tiene nombre existe. Mirentxu decide no hablar de la guerra, ni del hambre, no quiere mencionar la maldad y el sufrimiento que ve alrededor, hasta que en medio del bombardeo grita pidiendo ayuda a su madre (Fig. 5).

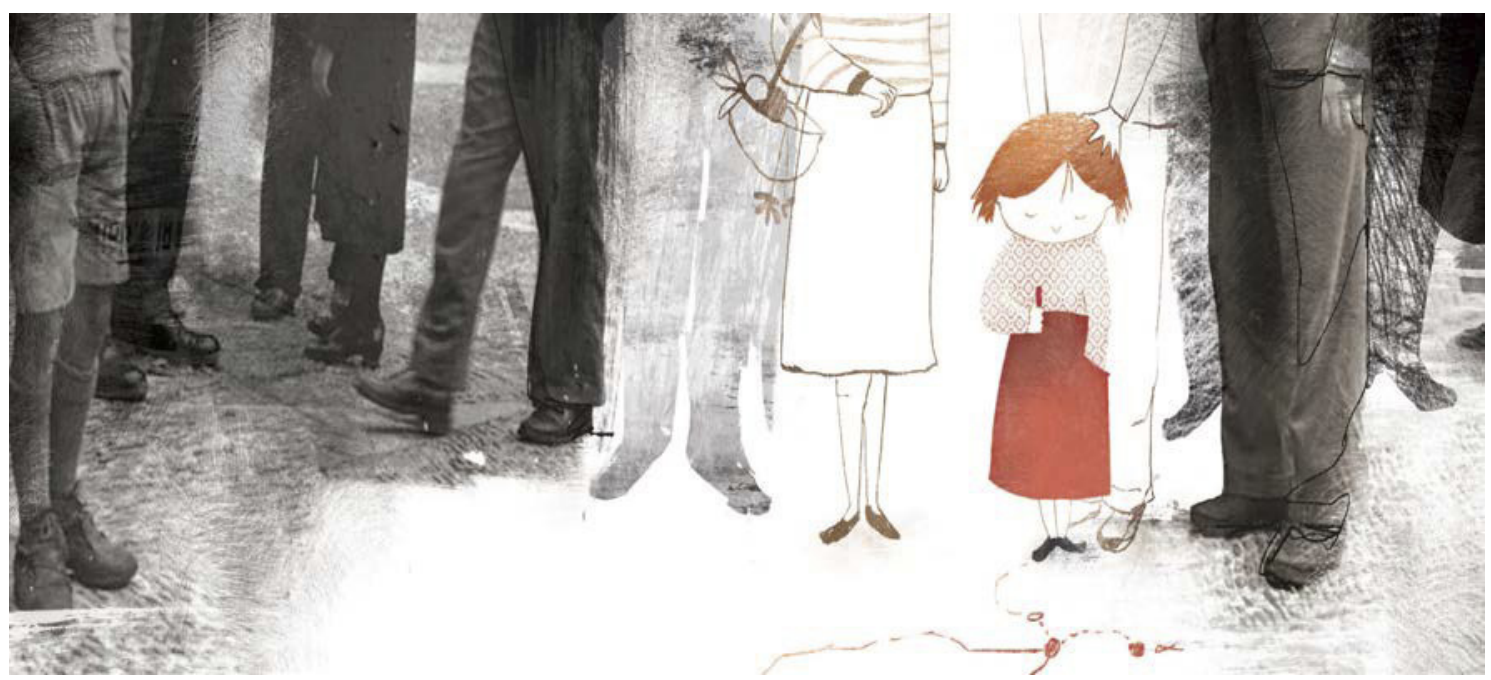

Fig. 5. Maite Mutuberria. Gerrak ez du izenik. Pamiela, Pamplona, 2013. 
El cuento y el álbum: (in)visibilidad femenina en la literatura infantil y juvenil vasca del siglo XXI

Sin duda estos modelos ayudan a formar una imagen más positiva de las mujeres, una imagen que contribuya a que sean más activas, sujetos creadores de su propio futuro y con su propio espacio; una mujer que rompe con el estado de dependencia que criticaban hace unas décadas pensadoras como S. De Beauvoir (1981) y, que hoy en día se sigue manteniendo en ciertos niveles (Fernández, 2017).

Pero tal vez lo más llamativo, en cuanto a equiparación o igual entre géneros, lo apreciemos mejor en aquellas obras que además de dicha equiparación buscan romper con todo tipo de estereotipos (Fig. 6).

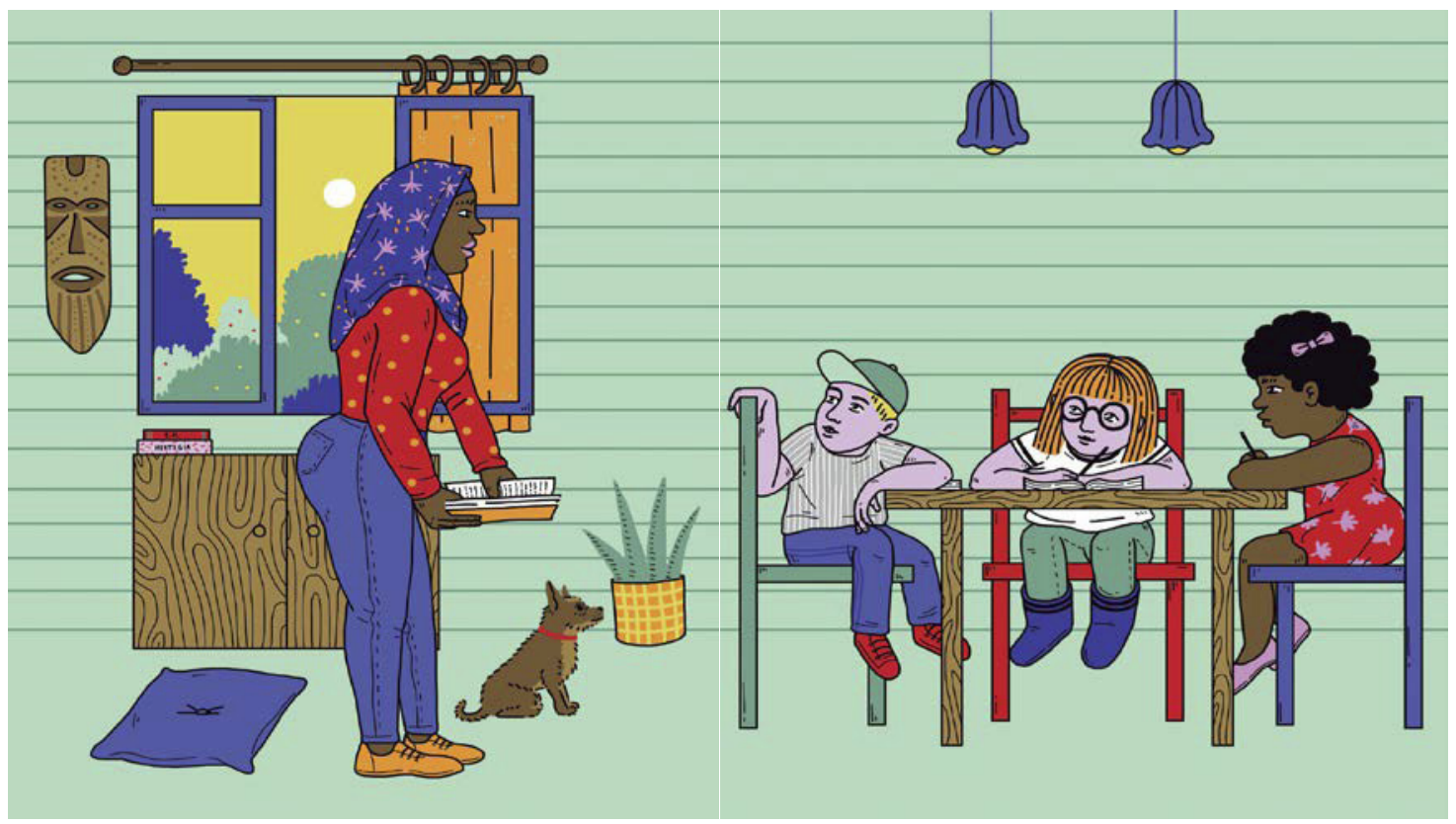

Fig. 6. Ainara Azpiazu. Arraroa. Pamiela, Pamplona, 2017.

Así, en el álbum Arraroa, Ainara Azpiazu y Paula Estévez nos presentan a un señor que pone su peluca rubia a tender y se pinta para salir de noche, una madre negra que les ayuda en las tareas de casa a los niños del bloque, estudiantes que no se sabe quién es chico y quién chica por la pinta que tienen, o la abuela que, aunque esté siempre en la cama, viaja con su nieta a través de los juegos. Porque en este libro lo que se nos muestra es que todos somos distintos, que a fin de cuentas todos somos iguales. Y de la misma manera que debemos poner el foco en la imagen de la mujer en la LIJ, otro tanto debemos hacer con la del hombre, puesto que lo que hace falta es un nuevo modelo de hombre en los libros infantiles. 


\section{Xabier Etxaniz}

Dicen García Villanueva y Hernández Ramírez (2016, p. 94) que «Los hombres y las mujeres no llegan al mundo programados para ser o actuar de una determinada manera, desarrollan capacidades según las condiciones y oportunidades que el medio les ofrezca.» En este sentido, creemos que es clave la labor de la LIJ para crear determinadas condiciones y oportunidades, para ofrecer una nueva visión del hombre y de la mujer actual, para contribuir al cambio de la sociedad en pos de una sociedad más igualitaria (Fig. 7).

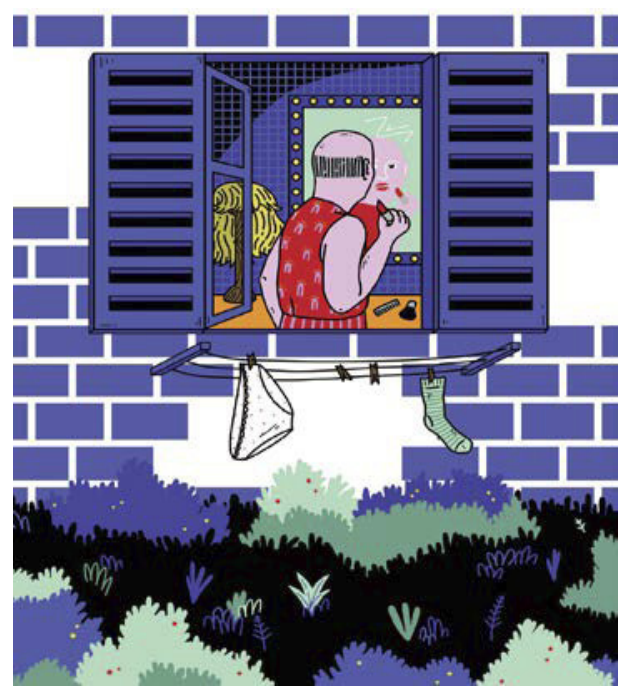

Fig. 7. Ainara Azpiazu. Arraroa. Pamiela, Pamplona, 2017.

Indicaban Colomer y Olid (2009) que parece como si se hubiese abandonado la batalla en pos de la igualdad que «se creyó ganada de forma irreversible». Está claro que, aunque en algunos aspectos se han dado pasos positivos, por desgracia queda mucho camino por recorrer y en este recorrido podemos contar con la aportación de los álbumes para visibilizar a las mujeres del siglo XXI.

\section{Referencias bibliográficas}

Almagia, K. (2009). La transmisión de valores en los cuentos. Revista Emakunde 75, 18-21.

Añorga, P. (2006). Gerlari handia. Iruñea: Pamiela.

Añorga, P. (2015). Munstroek ere pixa egiten dute ohean. Arre: Pamiela.

Asiain, M. (2011). Hara! Iruñea: Pamiela.

Azpiazu, A. (2017). Arraroa. Arre: Pamiela. 
El cuento y el álbum: (in)visibilidad femenina en la literatura infantil y juvenil vasca del siglo XXI

Bilbao, L. (2013). Gerrak ez du izenik. Iruñea: Pamiela.

Colomer, T.; Olid, I. (2009). Princesas con tatuaje: las nuevas caras del sexismo en la ficción juvenil. Textos de Didáctica de la Lengua y la Literatura, 51, 55-67. Recuperado de http://www.gretel. cat/wp-content/uploads/2016/01/Princesitas.pdf. Última consulta: 20-1-2019.

De Beauvoir, S. (1981). El segundo sexo. Madrid: Aguilar.

Etxaniz, X. (1997a). Euskarazko haur eta gazte literatur ilustratzaileak - Ilustradores de literatura infantil y juvenil. Vitoria-Gasteiz: Arabako Foru Aldundia-Diputación Foral de Álava.

Etxaniz, X. y Olaziregi, M. (1997b). Euskarazko Haur eta Gazte Liteatura Idazleak-Escritores de literatura infantil y juvenil en Euskara. Pamplona: Pamiela.

Etxaniz, X. (2013). Hacia la feminización de la literatura infantil, INSULA 797, 12-14.

Etxaniz, X. (2017). Una literatura infantil y juvenil más amplia y feminizada, in Anuario Iberoamericano sobre el Libro Infantil y Juvenil 2017 (pp. 281-293). Madrid: Fundación S. M.

Fernández, E. (2017). Emakumearen irudia euskal haur literatura modernoan. 1980ko hamarkadako eta gaur egungo egoera. Bilbo: UPV/EHU. Tesis doctoral.

Freixas, L. (2009). La novela femenil y sus lectrices. Córdoba: Servicio de publicaciones, Universidad de Córdoba.

García Villanueva, J. y Hernández R., C. I. (2016). ¿Realidad o fantasía? Roles y estereotipos sexistas expuestos a través de representaciones discursivas e iconográficas en cuentos infantiles. Revista Integra Educativa Vol IX nº1. 91-110.http://www.scielo.org.bo/scielo. php?pid=S1997-40432016000100006\&script=sci_arttext Fecha de la última consulta: 3 octubre 2018.

Gurrutxaga, M. (2009). Alex nire laguna. Iruñea: Pamiela.

Martínez, I. (2016). Zerua gris dago. Arre: Pamiela.

Martirena, D. (2018) Cosimoren katiuskak. Arre: Pamiela.

Moreno, A. (1998). Literatura infantil: Introducción en su problemática, su historia y su didáctica. Cádiz: Universidad de Cádiz.

Ormzabal, J. (2007). Zirkua amets. Iruñea: Pamiela.

Salaberria, L. (2010). Euria ari duenean. Iruñea: Pamiela. 


\section{Xabier Etxaniz}

Salaberria, L. (2014). Hiltzaileak. Iruñea: Pamiela.

Schweickart, P. (1999). Leyendo(nos) nosotras mismas. Hacia una teoría feminista de la lectura. In Fe. M. (coord..) Otramente, lectura y escritura feministas (pp. 112-151). Madrid: Fondo de Cultura Económica.

Turin, A. (2009). El poder de las ilustraciones. Revista Emakunde 75, 30-33.

Vázquez García, C. (Ed.) (2010). Diálogos Intertextuales 3: En busca de la voz femenina. Temas de género en la literatura infantil y juvenil de la Península Ibérica y Latinoamérica. Frankfurt: Peter Lang

Zubeldia, I. (2008). Katalina kontalari. Iruñea: Pamiela.

\section{Anexo I}

LISTADO ESCRITORES/AS GALARDONADAS CON EL PREMIO LIZARDI DE LIJ 2000-Begoña Bilbao

2001-Karlos Zabala

2002-Imanol Azkue

2003-Desierto

2004-Pello Esnal

2005-Imanol Azkue

2006-Desierto

2007-Rubén Ruiz

2008-Xabier Mendiguren

2009-Castillo Suárez

2010-Arrate Egaña

2011-Idoia Etxeberria

2012-Oihane Fernández

2013-Ramón Olasagasti

2014-Alaine Agirre

2015-Nerea Arrien

2016-Leire Bilbao 
El cuento y el álbum: (in)visibilidad femenina en la literatura infantil y juvenil vasca del siglo XXI

2017-Ramon Olasagasti

\section{Anexo II}

LISTADO ESCRITORES/AS GALARDONADAS CON EL PREMIO EUSKADI DE LIJ

2000- Felipe Juaristi

2001-Karlos Linazasoro

2002-Miren Agur Meabe

2003-Fernando Morillo

2004-J.M. Olaizola, $<<$ Txiliku $>>$

2005-Xabier Olaso

2006-Patxi Zubizarreta

2007-Miren Agur Meabe

2008-Pello Añorga

2009-Rubén Ruiz

2010-Patxi Zubizarreta

2011-Miren Agur Meabe

2012-Iban Zaldua

2013-Xabier Olaso

2014-Harkaitz Cano

2015-Yolanda Arrieta

2016-Uxue Alberdi

2017-Leire Bilbao.

2018-Eider Rodríguez

Anexo III

LISTADO ILUSTRADORES/AS GALARDONADAS CON EL PREMIO EUSKADI DE ILUSTRACIÓN

2009-Elena Odriozola

2010-Jokin Mitxelena 


\section{Xabier Etxaniz}

2011-Iban Barrenetxea

2012-Sara Morante

2013-Elena Odriozola

2014-Maite Gurrutxaga

2015-Ana G. Lartitegui

2016-Mikela Casal

2017-Mikel Valverde

2018-Sara Morante

\section{Anexo IV}

\section{LISTADO DE OBRAS GANADORAS DEL PREMIO ETXEPARE}

Añorga, P. / Jokin Mitxelena (2006). Gerlari handia. Iruñea: Pamiela.

Ormazabal, J. / Iraia Okina (2007). Zirkua amets. Iruñea: Pamiela.

Zubeldia, I. / Estibaliz Jalon (2008). Katalina kontalari. Iruñea: Pamiela.

Gurrutxaga, Mikel / Maite Gurrutxaga (2009). Alex nire laguna. Iruñea: Pamiela.

Salaberria, Leire (2010). Euria ari duenean. Iruñea: Pamiela.

Asiain, Miren (2011). Hara! Iruñea: Pamiela.

Bilbao, Leire / Maite Mutuberria (2013). Gerrak ez du izenik. Iruñea: Pamiela.

Salaberria, Leire. (2014). Hiltzaileak. Iruñea: Pamiela.

Añorga, Peio / Jokin Mitxelena. (2015). Munstroek ere pixa egiten dute ohean. Arre: Pamiela.

Martínez, Isaak / Liebana Goñi. (2016). Zerua gris dago. Arre: Pamiela.

Azpiazu, Ainara / Paula Estévez. (2017). Arraroa. Arre: Pamiela.

Dani Martirena / Ana Ibáñez (2018) Cosimoren katiuskak. Arre: Pamiela. 